\section{Military Medicine and Surgery}

\section{$\triangle$ NEW ADAPTATION OF THE FACE MASK IN CONTROL OF CONTAGIOUS DISEASE}

\author{
JOSEPH A. CAPPS, M.D. (ChICAGo) \\ Major, M. R. C., U. S. Army \\ CAMP GRANT, ROCKFORD, ILL.
}

One of the most troublesome problems confronting the medical staff of the base hospitals in camps is the prevention of cross infections.

$\Lambda$ mistaken diagnosis in the examining room may place measles in a scarlet fever ward, or meningitis in a clean diagnostic ward. These mistakes are partially remedied by sending all doubtful patients to an observation ward, where they may be differentiated at lcisurc.

The development of a secondary infection as a result of an exposure some time previous to entrance into the hospital is unavoidable.

On the other hand, the contraction of a secondary infection directly from another individual in the same ward demands the most serious effort in the way of prevention. When a case of scarlet fever breaks out in a measles ward, the ward must be placed in quarantine for a week. By the end of the week another case may develop and require another week of quarantine. This may go on indefinitely so that the ward is barred from either receiving or discharging patients.

Most contagious diseases, such as measles, German measles, scarlet fever, pertussis, meningitis and diphtheria, are transmitted from one person to another by means of the secretions of the nose and mouth. Coughing, sneezing and talking convey the bacterialarlen droplets of mucus through the air.

The cubicle system, now used extensively in the Army hospitals, and consisting of sheets suspended on wire between the beds, restricts the dissemination of droplets so long as the patients remain in bed.

In addition to this system we have carried out from the beginning, at the Camp Grant base hospital, the practice of requiring physicians, nurses and ward men to wear gowns and gauze face masks. In this we have followed as closely as possible the methods employed at the Durand Hospital in Chicago. Weaver, ${ }^{1}$ in a recent report, shows the efficacy of the mask at the Durand Hospital in protecting the physicians and nurses from contracting contagious diseases and from becoming carriers of meningococci and diphtheria hacilli. We can also attest to the remarkable protection of the mask at Camp Grant, for during the first five months not one nurse or physician contracted the disease of the patients with whom he or she was working. With all these measures, however, we were unable to prevent the spread of cross infection from patient to patient, since we have no facilities for effectively separating patients in a ward once exposed to a fresh contagious disease. The cubicle segregates the bed patients, but it is almost impossible to confine convalescent soldiers day and night to bed; furthermore, it is not possible to furnish the necessary service in nursing, bathing and feeding to render such segregation effective. The patients use a common wash room

1. Weaver, G. H.: The Value of the Face Mask and Other Measures, TuE Journal A. M. A., Jan. 12, 1918, p. 76. and latrine, and hence the opportunity of transmission is unlimited.

The quarantine of several measles wards at a time by successive outbreaks of scarlet fever created at Camp Grant, last winter, an acute situation. To relieve this situation we carried out an experiment, the description of which is my purpose in this preliminary paper.

\section{MASKING THE PATIENT}

The face mask has proved an undoubted success in protecting physicians and nurses from contagion. It seems reasonable that patients by wearing the mask should receive similar protection from either primary contagion, or if already ill with one infection, from a second contagion.

The experiment of masking the patients was first tried in a measles ward in which six successive cases of scarlet fever had developed at intervals of several days. After the introduction of this method, one more case of scarlet fever broke out in five days, but none subsequently. The system has since been tried in other wards with excellent results. In general wards exposed to scarlet fever the masks are worn by all patients for one week after the last exposure.

The essential principle of the method is that patients out of bed and out of the cubicle must never be unmasked, except when alone in the wash room. In order to make it effective a number of precautions a re necessary. The following rules are posted in each ward and latrine:

\section{RULES FOR CONTAGIOUS WARDS}

1. A face mask must be worn continuously by all paticnts when out of cubicle.

2 . In the latrine the mask may be removed only by permission and under the direct supervision of ward nurse.

3. Wash basins and bath tub are not to be used. For washing face and brushing teeth use running water over sink. Shower may be used under superviuion. Use only liquad soap from container.

4. Only one patient will be allowed in the wash room at one time. Remove mask on entering and replace before leaving wash room.

5. Masks may be removed when patients are in bed.

6. Sheets between beds are not to be drawn back.

7. No smoking allowed.

8. Corps men, nurses and surgeons should wear masks and gowns when on duty in the ward. A guard should be on duty continually near the wash room.

9. All eating utensils should be sterilized after each meal.

MASKING PATIENTS IN THE AMBULANCE

Many patients in the hospital break out with a fresh contagious disease, the incubation period of which corresponds to the length of time they have been in the hospital. This circumstance strongly suggests that the disease was contracted on the date of admission. As patients with different infections frequently come to the hospital together in the same ambulance, the chance of cross infections is obvious. Attempts to avoid this danger by grouping cases affected with the same disease have been futile, because many are sent to the hospital at the beginning of fever or eruption and are not yet diagnosed. Further exposure to contagion takes place in the admission room, where patients are often crowded together awaiting examination.

To guard against this danger we have made it a rule that every patient before entering the ambulance shall put on a face mask and continue to wear it until he 
reaches his bed in the ward. This applies to all patients, whether they have any communicable disease or not.

It is too early to say to what extent this masking of patients in the ambulance has prevented cross infections, but we believe it has lessened the number materially.

\section{AN ANALYSIS OF GOVERNMENT CARDIO- VASCULAR EXAMINATIONS}

AS CONDUCTED AT THE BASE HOSPITAL, CAMP HANCOCK, AUGUSTA, GA.

\section{S. CAlvin SMith, M.D. (Philadelphia)}

Captain, M. R. C., U. S. Army

CAMP CUSTER, BATTLE CREEK, Mich.

These statistics have been compiled from the cardiovascular examinations of members of the TwentyEighth Division, N. G., recently completed. The totai strength of the command approximated 35,000 men, of which number 1.11 per cent. were recommended for discharge, which recommendations form the basis of this analysis. While absolute statistics are of course not available, owing to the constantly changing personnel of the division, sufficient data from the cardiac examinations are available to form a figure basis for this report.

Familiar methods of physical examination were employed, plus the instrumental aid of the stethoscope, sphygmomanometer and polygraph. It is to be regretted that electrocardiography was not available, especially for the information which it would have afforded in differentiating the tachycardias, which may have been due to vagal or sympathetic nerve influence and hence extracardiac in origin, or which may have arisen from irritable or degenerative foci within the auricular or ventricular muscle. Further, the written record obtained from electrocardiography in borderline or in doubtful cases would be of profound statistical importance for future heart recruiting standards, when considered in connection with the subsequent sick reports of such men who are now accepted for service.

\section{THE RECORDING OF EXAMINATIONS}

A blank, the form of which is given herewith, was filled out for each man examined. The individual records were made available for future reference by cross-indexing the serial number under the headings "Patient's Index" and "Diagnosis Index." In order to facilitate the work of the clerk who recorded the findings as called off by the examining physician, certain standard descriptive words were printed on the blank that required only an underscoring stroke of the pen to record. The form is self-explanatory.

\section{BASIS FOR REJECTIONS}

The principles of interpretation of cardiac disease, as laid down in Circular 21, Surgeon-General's Office, July 14,1917 , were followed so far as any set of rules could govern the individual case being considered. Further than this, visible pulsations which were not due to emotional disturbance, accelerated pulse rates not attributable to the same cause, maximum cardiac impulses displaced downward or to the left, and any changes in rate, rhythm or volume of the pulse, were all accorded more significance than were the detec- tions of cardiac murmurs, many of which disappeared on recumbency or following exercise. This most unconventional disappearance of physiologic or functional murmurs following exercise was attributed to a decreased tonicity of a strong heart when at rest ; exercise increased the tonicity, the relaxed valve leaflets

\section{RECORD OF ORIGINAL EXAMINATION}

Serial No...............

Cardiovascular Department, Base Hospital

Camp Hancock, Augusta, Ga.

Date.........................

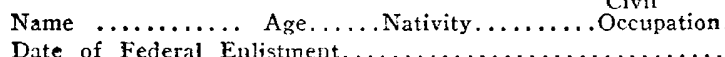

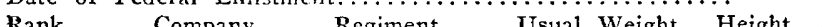

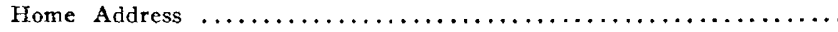

Reason Referred

By Whom Referred.

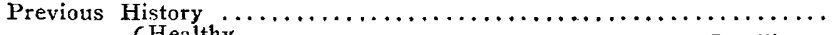

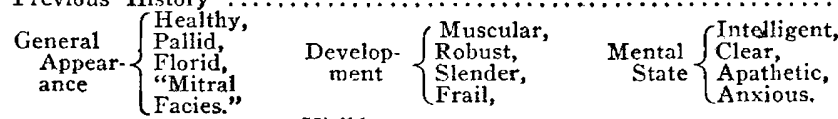
$\begin{array}{ll}\text { Facies." } & \text { Visible } \\ \text { Pulsations, Edema....... } & \text { Pretibial } \\ \text { Sacral, } & \text { Present: } \\ \text { History of }\end{array}$

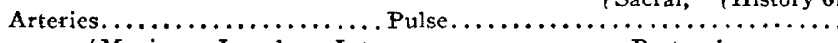
Apex: $\{$ Maximum Impulse... Int.

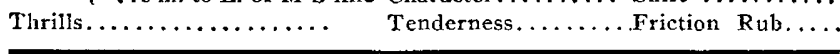

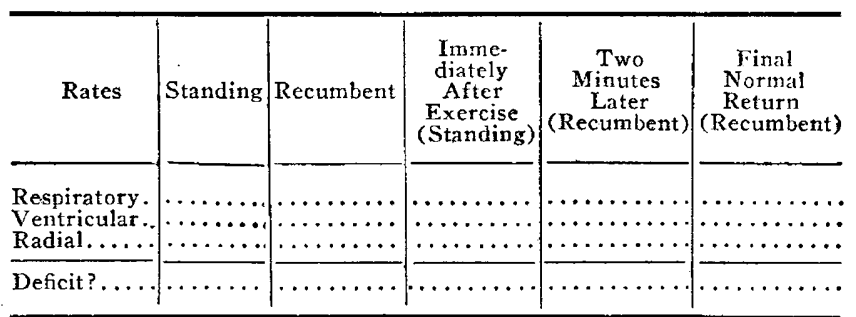

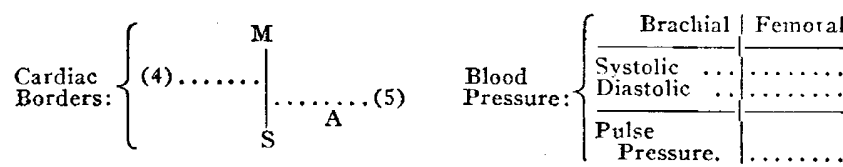

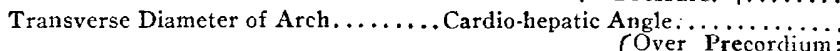
(Apical Systolic, Presys- $\quad$ Over Precordium: Murmurs:\{Apical $\left\{\begin{array}{l}\text { Systolic, Presys- } \\ \text { tolic: Diastolic: Transmission: }\end{array}\right.$ To Axilla: To Back.

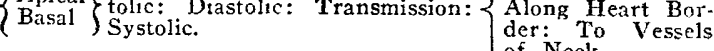

Accentuations: A. .; T..; P..; M. .; Reduplication................ Arrhythmia $\left\{\begin{array}{l}\text { None. } \\ \text { Disappears on Exercise. Graphic Record. } \\ \text { Increases on Exercise. }\end{array}\right.$

Clinical Tests: Wassermann......... Roentgen Ray ..............

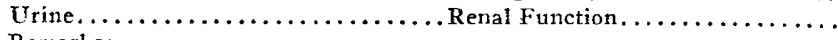

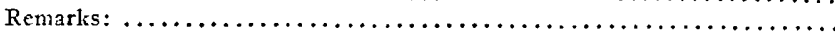

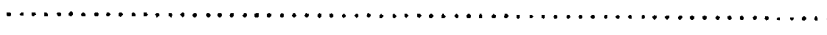

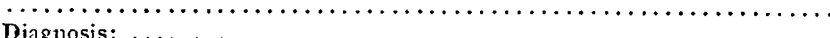
Recommendation:-(1)-Acceptance. (2)-Discharge. (3)-Acceptance for Special Service. (Cross out two)

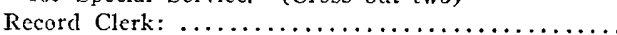

Reviewed and approved by Dr. S. Calvin SMtTh,

In Care of Cardiovascular

came again into apposition, and the murmur consequently disappeared. Full significance was of course accorded to a murmur which persisted at a given area and which was accompanied by accentuations of other sounds, provided it was transmitted along the usual classical lines for a given murmur, or accompanied by the characteristic thrills, by increased transverse diameters of given chambers of the heart, or by other frank evidences of cardiac disease.

The exercise test of hopping 100 times on one foot yielded most valuable information, when taken in connection with other physical signs; it frequently elicited testimony of cardiac hypertrophy and myocardial 